ISSN: 2600-5859

\title{
Estenosis de Anastomosis Hepático-yeyunal Termino- Lateral en Y de Roux tratada mediante reconfección y modificación de la técnica de Anastomosis a Termino- Terminal
}

Estenosis de Anastomosis Hepático-Yeyunal Termino- Lateral en Y de Roux tratada mediante reconfección y modificación de la técnica de Anastomosis a Termino-Terminal

Fanny Karina León Loaiza. ${ }^{1}$, Diego Medardo Andrade Astudillo. ${ }^{2}$

Recibido:01-12-2019 / Revisado: 02-01-2020 /Aceptado: 18-01-2020/ Publicado: 07-02-2020

\section{Resumen DOI: https://doi.org/10.33262/concienciadigital.v3i1.1.1148}

Las estenosis tempranas de la vía biliar luego de una anastomosis bilio-digestiva se relacionan con aspectos del procedimiento quirúrgico mientras que las tardías con fenómenos inflamatorios, fibrosis por fugas biliares, isquemia por lesión vascular, reflujo yeyuno-biliar o colangitis séptica por fenómeno de asa ciega; el tratamiento definitivo es una nueva Hepático-yeyuno anastomosis. El presente caso se trata de un paciente de 39 años de edad intervenido por lesión de vía biliar Bismuth 4 ocasionada durante una colecistectomía laparoscópica, se lo trato mediante Hepático-yeyuno-anastomosis Termino-lateral con Y de Roux después de la cirugía presentó episodios de colangitis a repetición por lo que fue referido al Hospital Pablo Arturo Suárez de Quito donde se resolvió el cuadro mediante la modificación de la técnica quirúrgica de anastomosis Termino-lateral a Termino-terminal procedimiento que se realiza en los pacientes atendidos en este hospital por haber demostrado menor morbilidad posoperatoria en comparación con la técnica clásica.

Palabras Clave: Derivación bilio-digestiva, Lesión iatrogénica biliar, cirugía del conducto biliar.

1 Universidad Nacional de Loja, Facultad de la Salud Humana, Loja, Ecuador, email: fanny.leon@unl.edu.ec

2 Departamento de Cirugía del Hospital Pablo Arturo Suárez, Quito, Ecuador, mail: medardodiegp@gmail.com 


\begin{abstract}
Early biliary stenosis following a biliary-digestive anastomosis is related to the surgical procedure, while late stenosis with inflammatory phenomena, biliary leakage fibrosis, vascular injury ischemia, jejuno-biliary reflux, or septic cholangitis due to the wing phenomenon blind. The definitive treatment is a new liver-jejunal anastomosis. The present case involves a 39 year old patient who was operated on for the bismuth 4 bile duct injury caused during laparoscopic cholecystectomy treated by Liver-jejunum-anastomosis Termino-lateral with Roux-en-Y after surgery presented cholangitis episodes a repeat for that reason was referred to the Hospital Pablo Arturo Suárez de Quito where the case was resolved by modifying the surgical technique of anastomosis Termino-lateral to Termterminal procedure that is performed in patients treated in this hospital for having demonstrated lower Postoperative morbidity compared to the classical technique.
\end{abstract}

Keywords: Biliodigestive derivation, iatrogenic biliary injuries, bile duct surgery.

\title{
Introducción
}

La lesión iatrogénica de la vía biliar (LIVB) se define como aquella lesión ocasionada en alguna parte de la vía biliar extra hepática durante la colecistectomía u otros procedimientos invasivos. Se considera que los factores de riesgo más comunes son las variaciones anatómicas, errores de percepción anatómicas de la vía biliar o por un proceso inflamatorio agudo o subagudo, sangrado o falta de experiencia del cirujano. "Su frecuencia varía en diferentes reportes entre 0,18\% a 0,5\% siendo dos veces más común la laparoscópica 0,3 abierta vs 0,6 laparoscópica” (Velasco, 2018, p.160). Las connotaciones de esta complicación son muy preocupantes pueden variar desde fuga de conductos secundarios hasta la sección completa del árbol biliar extra hepático la misma que puede acompañarse de lesión vascular; en estos casos la solución es compleja por lo que el manejo debe ser multidisciplinario en centros de referencia con cirujanos expertos en el manejo de estas lesiones. El tratamiento de la LIVB puede ser quirúrgico, endoscópico, radioscópicos o mixto dependiendo del tipo de lesión según la clasificación que se utilice de Strasberg o Bismuth. "La Hepático yeyuno anastomosis en Y de Roux (HYA/YR) es el tratamiento estándar para la mayoría de las lesiones de la vía biliar post colecistectomía, con tasas de éxito clínico a largo plazo que alcanzan el 90\%” (Benkabbou, 2013, p.1). Por lo cual este procedimiento ha sido el más aceptado por los cirujanos expertos para la resolución de las lesiones complejas Strasberg E4 y E5 o (Bismuth 4 y 5). El abordaje que se realiza generalmente es el tratamiento quirúrgico abierto, 
Pacheco (2016) afirma en su estudio: “La reparación de vía biliar se realizó mediante una laparotomía en el 97\% de los pacientes. Es la vía utilizada en la mayoría de las series nacionales e internacionales. La vía laparoscópica se ha comenzado a usar en casos altamente seleccionados y en centros con experiencia en cirugía laparoscópica avanzada, (...). La tasa de complicaciones que se puede presentar luego de la reparación puede variar 32 y 37,5\%.” (p.205)

Las complicaciones que se pueden presentar luego de una HYA pueden ser por estenosis a nivel de la anastomosis 9 a 25\%, hipertensión portal 15 a 20\%, cirrosis de la vía biliar $8 \%$ y colangitis. En el caso de una estenosis el tratamiento precoz es la Colangiografía transparieto-hepática (CTPH) y drenaje trans hepático para solucionar la colangitis. Pero finalmente una nueva HYA es la solución más adecuada a corto y largo plazo. (Ruiz, 2019, p.216-217).

Como ya se mencionó el manejo de LIVB debe ser por un centro especializado en manos de cirujanos expertos quienes decidirán el mejor tratamiento según el tipo de la lesión biliar clasificación de Strasberg o Bismuth, ante este problema que puede acontecer si no se tiene la experticia y condiciones adecuadas para la cirugía de vía biliar, los cirujanos especialistas en reconstrucción de vía biliar coindicen en que la técnica definitiva para la resolución de la complicación S4-S5 o B4 es la Hepatico-Yeyuno Anastomosis en Y de Roux termino lateral, exceptuando los casos extremos y fallidos en los que se puede requerir trasplante hepático.

En el presente caso clínico se mencionan modificaciones de la técnica de anastomosis como parte del procedimiento quirúrgico para obtener mejores resultados para los pacientes. La especial importancia en el procedimiento quirúrgico de reparación es tan importante como su seguimiento para la obtención de resultados satisfactorios en términos de calidad de vida del paciente mediante una disminución de la demanda de morbilidades relacionadas con las complicaciones de la cirugía de reparación de vía biliar.

\section{Descripción del caso clínico}

Paciente de sexo masculino de 39 años de edad sin antecedentes clínicos de importancia como antecedentes quirúrgicos presenta Colecistectomía Laparoscópica por Colelitiasis durante la cual se produjo una lesión de vía biliar posterior a la misma tuvo que ser sometido a una HAY en Y de Roux Termino-Lateral, un mes después de la cirugía. Posteriormente es referido al HPAS por presentar cuadros recurrentes de obstrucción biliar el último episodio se presentó 5 días previos al ingreso del paciente quien refirió dolor abdominal continuo en epigastrio e hipocondrio derecho, 
ISSN: 2600-5859

Vol. 3, N¹.1, p. 284-295, febrero, 2020

anorexia, alza térmica no cuantificada. Al ser examinado presentó escleras ictéricas, abdomen doloroso en epigastrio e hipocondrio derecho ruidos hidroaeéreos normales. Los exámenes de laboratorio realizados: biometría hemática que presenta neutrófilos elevados (70.5\%), Fosfatasa Alcalina de 860, GGT: 869, TGP: 112. La Colangioresonancia (CRM) evidencia dilatación de vías biliares intrahepáticas e imagen sugestiva de estenosis en el sitio de anastomosis hepático yeyunal.

\section{Figura 1}
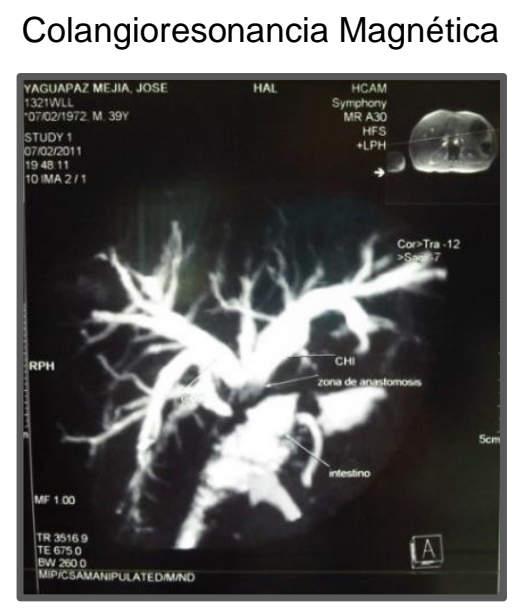

Fuente: Fotografía de la CRM del paciente

Se realizó nueva Hepático-yeyuno anastomosis con técnica Termino-Terminal en Y de Roux, colocación de sonda Kehr y drenaje tubular aspirativo subhepático.

\section{Figura 2}

Identificación de lesión Bismuth IV luego de desmontar Anastomosis

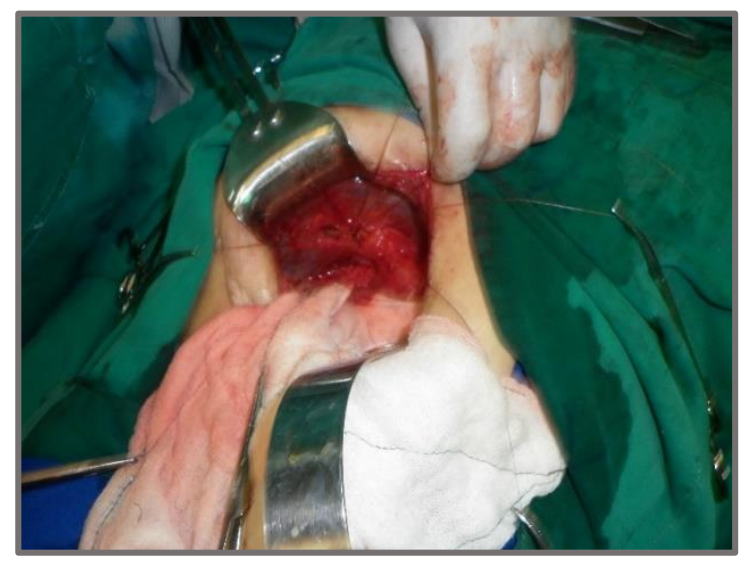

Fuente: Fotografía de la Cirugía del paciente 


\section{Figura 3}

Colangioscopía durante la cirugía.

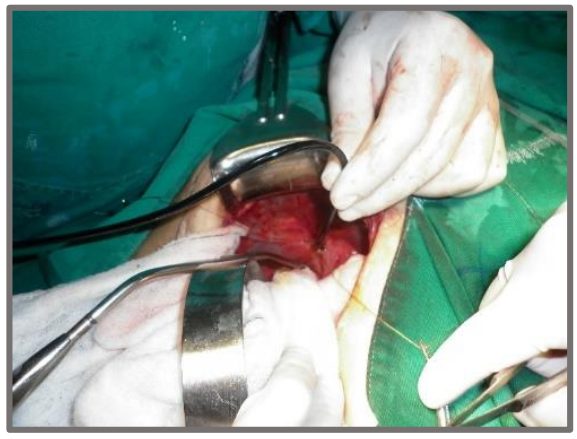

Fuente: Fotografía de la Cirugía del paciente

\section{Figura 4}

Colocación de Sonda Kehr

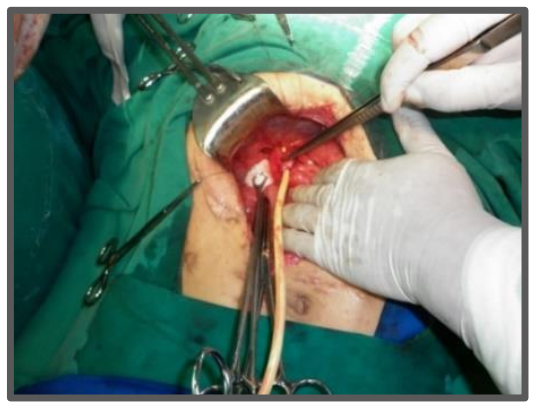

Fuente: Fotografía de la Cirugía del paciente

\section{Figura 5}

Asa para la Y de Roux.

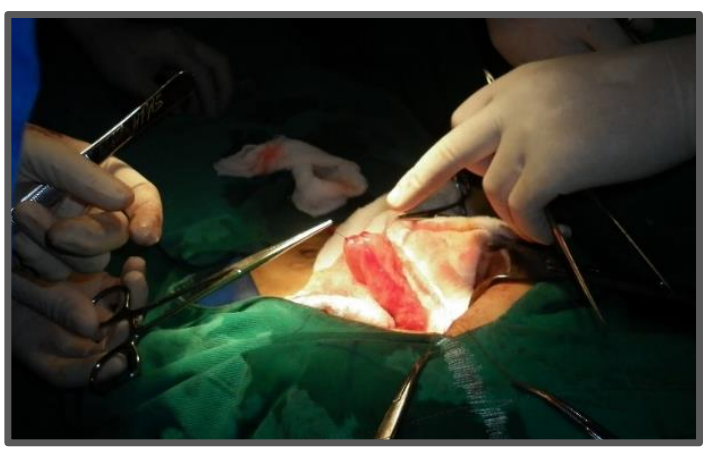

Fuente: Fotografía de la Cirugía del paciente 
Los hallazgos de la cirugía fueron: estenosis casi total de la anastomosis, conductos hepáticos separados equivalente a una lesión Bismuth IV, cálculos que obstruían la luz y contenido purulento. Se realizó Colangioscopía en la cual se evidencio los conductos hepáticos con múltiples estigmas de cálculos y pus. Asa en Y de Roux con luz adecuada.

El Procedimiento quirúrgico consistió en una ampliación de la boca anastomótica mediante plastia de la confluencia de los conductos hepáticos y por medio de incisiones en la cara anterior de los conductos cerca de 5mm. Nueva HYA Termino-Terminal con Y de Roux asa desfuncionalizada a $60 \mathrm{~cm}$ de longitud, colocación de sonda Kehr abocada de a $1.5 \mathrm{~cm}$ de la anastomosis hepáticoyeyunal y drenaje tubular aspirativo subhepático.

En el posoperatorio el paciente tolera líquidos a las 48 horas, la sonda Kehr con producción de líquido biliar de $250 \mathrm{ml}$ el día del alta médica, el drenaje tubular aspirativo con producción de líquido hemático 24 horas pos operatorio luego sero-hemático. Es enviado de alta en buenas condiciones con la sonda Kehr. Se realiza Colangiografía trans Kehr a los 8 días evidenciándose permeabilidad de vías biliares. A los 30 días posoperatorio se cierra la sonda Kehr y se indica la instilación de $10 \mathrm{ml}$ de solución salina al 0,9\% tres veces al día para evitar precipitaciones y colonización del Kehr, el cual se mantiene hasta que en los controles colangiográficos mensuales, no haya evidencia de re estenosis, se haya desplazado o ya no cumpla su función. Para el retiro del Kehr en los pacientes tratados en este hospital no se toma como referencia el tiempo transcurrido.

\section{DISCUSION}

La identificación y resolución de una LIVB se debe hacer de manera temprana durante el procedimiento quirúrgico determinando el tipo de lesión, la situación clínica del paciente, la presencia de lesiones vasculares asociadas y los factores hospitalarios. En el presente caso la LIVB fue identificada en el posoperatorio de manera tardía debido a que el paciente presentó síntomas de colangitis luego de la cirugía los cuales fueron: dolor abdominal tipo cólico de moderada intensidad, alza térmica e ictericia los exámenes de laboratorio corroboraron hiperbilirrubinemia a expensas de la bilirrubina indirecta, así como elevación de las transaminasa y gama glutamil transpeptidasa también se realizó la Colangioresonancia magnética determinándose en primera instancia la lesión iatrogénica de la vía biliar Bismuth 4 por lo que se decidió realizar una Derivación Bilio-Digestiva (DBD) desafortunadamente un mes después presento el mismo cuadro de colangitis el cual se confirmó por Colangioresonancia en la cual se 
observa una clara estenosis de la anastomosis. El diagnóstico tardío de la lesión al igual que el tipo de lesión probablemente influyo en la aparición temprana de estenosis de la anastomosis teniendo que referirse al paciente a otro centro terciario donde se resolvió la patología con buenos resultados demostrándose por una estancia hospitalaria corta y mejoría clínica significativa.

El estado clínico del paciente y el tiempo de reparación de la lesión ya sea en etapa temprana o tardía influyen en la morbilidad y mortalidad del paciente. Hishaam (2016) afirma "el grado de riesgo quirúrgico determinado por ASA (American Society Anesthesiologists) así como las reparaciones tempranas en pacientes con colangitis aguda se asocian con resultados peores por lo que el autor recomienda un enfoque multidisciplinario en un centro especializado, controlar la infección y mejorar el estado funcional antes de la reconstrucción quirúrgica” (p.352). En este caso se presentó Colangitis la cual fue tratada antes del procedimiento ya que se inició terapia antimicrobiana pero existe diferencia en cuanto a la decisión de realizar una reparación en fase temprana ya que en este estudio no lo recomienda pero existen otros estudios que indican que "la reparación de la LIVB en etapas tempranas puede ser apropiada dependiendo de los factores que presenta el paciente en el caso de fuga de la anastomosis lo realizan de manera temprana mientras que en casos de estenosis dependiendo del grado de obstrucción o la presencia de colangitis" (Straka, 2017, p.90). En este caso se debe considerar que el paciente presentó una mala evolución, luego de una primera reparación tardía.

La aparición de colangitis en los casos de estenosis de anastomosis hepático-yeyunal obedecen a varios factores entre ellos el reflujo intestinal a la vía biliar por perdida del mecanismo de válvula, por presencia de cálculo en el sitio de la anastomosis o por la misma técnica quirúrgica que lleve a una estenosis y obstrucción. En casos de seguimiento posoperatorio realizados en pacientes con Anastomosis Bilio-digestiva (ABD) también se menciona que "la cirugía hepatobiliar da lugar a una pérdida del esfínter de Oddi con ascensión consecutiva de bacterias al sistema biliar que puede causar colangitis en el curso postoperatorio" "Camman. En este caso pudimos apreciar que luego de la primera anastomosis HYA realizada a los 30 días posteriores a la lesión se presentó la estenosis que posteriormente comenzó a dar repercusión en el paciente con síntomas de colangitis por lo que requirió una nueva re intervención y estabilización previa para la cirugía.

El tratamiento definitivo realizado en este paciente fue una Hepático-yeyuno anastomosis con técnica Termino-Terminal en Y de Roux y colocación de Sonda Kehr esta modificación en la 
técnica de anastomosis de termino-lateral a término-terminal se justifica por preservar la memoria celular al aumentar el perímetro del cabo biliar de los conductos hepáticos y disminuir la circunferencia de la luz del asa intestinal y así evitar la estenosis, el asa ciega y la colangitis a repetición lo cual se demostró en este paciente ya que presento resultados positivos demostrados por su mejoría clínica y estancia hospitalaria corta. La necesidad de mejorar los resultados obtenidos con la cirugía de DBD ha sido creciente en los cirujanos que realizan estos procedimientos. Hwang (2016) afirma "que la necesidad de hacer frente a situaciones difíciles como lesiones biliares complejas ya sea por lesión iatrogénica biliar o cáncer de las vías biliares son un reto; por lo que; idearon una alternativa a las técnicas convencionales de HYA con la denominada Hepático-jejunostomía en racimo $(\mathrm{JH})$, que puede ser acoplada con resección paliativa de vías biliares, la que consistió en la aplicación de varios stents internos biliares y una sola amplia porto-enterostomía a los tejidos conectivos circundantes esta técnica fue aplicada a un paciente con lesión biliar bilateral asociada a la colecistectomía laparoscópica con la cual no mostró complicaciones biliares en el período de 5 años posteriores a este procedimiento" (p.66). Con los resultados de este estudio, la técnica de $\mathrm{HJ}$ en racimo puede ser un método quirúrgico útil para la reconstrucción segura de los conductos biliares gravemente dañados. Con todo lo expuesto podemos tener una visión de cómo se puede realizar modificaciones a las opciones clásicas de tratamientos de vía biliar con las ABD según sea el caso de cada paciente, teniendo en cuenta que la técnica clásica descrita es la HYA-TL en Y de Roux para las lesiones Bismuto 4 y 5 o Starsberg E4 y E5 y que también ha sido muy aceptada en centros de referencia en manejo de lesiones de vías biliar, podemos citar a López (2015) quien en un estudio realizado en un Centro de referencia regional para patologías complejas y complicaciones hepatobiliares quirúrgicas indica que se demostró que los mejores resultados se obtuvieron con la HYA en Y de Roux.

En el caso presentado se realizó el mismo procedimiento, HAY en Y de Roux, pero con una modificación de la técnica quirúrgica realizando un aumento del diámetro del cabo biliar de la anastomosis y en la parte de la anastomosis haciéndola termino-terminal obteniéndose buenos resultados al reducir la morbilidad biliar asociada por el tipo de lesión que tenía el paciente.

En el HPAS de Quito sitio de referencia para resolución de LIVB establecimiento donde ser resolvió el presente caso clínico quirúrgico, también se buscó mejorar los resultados obtenidos con el procedimiento quirúrgico de $\mathrm{ABD}$ por lo que se ha implementado como la técnica más adecuada 
para tratamiento de LIVB la HYA Termino-Terminal con Y de Roux y colocación de Sonda Kehr. Realizándose en este hospital 30 HYA con los siguientes detalles: 11 con técnica clásica T-L que presentaron 5 re-estenosis (45\%) tratadas 2 con plastias, 2 re anastomosis TT y uno sin Tratamiento. Los otros 19 casos de LIVB fueron tratados con HYA Termino-Terminal con una reestenosis (5\%) tratada con una plastia y un caso con colangitis ( 2 episodios en 5 años) tratado con antibióticos. Demostrándose una disminución significativa de complicaciones en los pacientes tratados con la técnica Termino-Terminal con una significativa reducción en las tasas de morbilidad en los casos intervenidos ya que están presentes, pero en menor porcentajes.

Un detalle importante en el manejo de los pacientes intervenidos por LIVB con ABD es el seguimiento, en el estudio realizado por Booij (2018) sobre el seguimiento de pacientes sometidos a hepático-yeyunostomía a corto y largo plazo se realiza el seguimiento a corto plazo en < 14 días en 14-90 días y > 90 días y a largo plazo en un período de 10 años.

Similar a estos parámetros de seguimiento se corrobora el protocolo establecido en el HPAS cuyo seguimiento se realiza una vez al mes durante el primer año y luego una cita cada 3 meses. En estos últimos 10 años los pacientes tratados con HYA TT en Y de Roux han presentado baja morbilidad y mejoría de su calidad de vida, tal es así que el caso presentado ha tenido un seguimiento en un período de 7 años en los cuales el paciente ha sido controlado periódicamente manteniéndose clínicamente estable sin presentar complicaciones biliares que reduzcan su calidad de vida.

\section{CONCLUSIONES}

- El tratamiento adecuado en una LIVB requiere una detección temprana y corrección en manos de un cirujano con experiencia en lesiones de vía biliar.

- El tratamiento quirúrgico empleado influye notablemente en los resultados obtenidos por lo que se justifica buscar mejorar la técnica quirúrgica que aporte mayores beneficios funcionales.

- El uso de sonda Kehr en derivación bioliodigestiva ayuda a evitar la obstrucción y realizar controles posteriores.

\section{Digital}


ISSN: 2600-5859

\section{Referencias bibliográficas}

1. AC, A. (2012). Surgical reconstruction of post-cholecistectomy cicatricial biliary stenosis. PubMed, 99-104.

2. Benkabbou A, C. D. (2013). Tratamiento de la Hepaticoyeyunostomía en Y de Roux fallida. IntraMed, 95-102.

3. Cammann S1, T. K. (2016). Cholangitis in the postoperative course after biliodigestive anastomosis. PubMed, 715-724.

4. Cuendis-Velázquez, M.-C. A.-O. (2016). Laparoscopic hepaticojejunostomy after bile duct injury. Surgical Endoscopy, 30, 876-882.

5. Daniel Atl López Fabióla, A. G. (2015). Derivación Bilioentérica en un centro académico de referencia estatal en México. Cirujano General, 37, 82-90.

6. Ibrahim Abdelkader Salama, 1.,. (2014). Iatrogenic Biliary Injuries: Multidisciplinary Management in a Major Tertiary Referral Center. PubMed.

7. Ismael HN., C. S. (2017). The morbidity and mortality of hepaticojejunostomies for complex bile duct injuries: a multi-institutional analysis of risk factors and outcomes using NSQIP. PubMed.

8. José Cooke, L. A. (2014). LESIONES QUIRURGICAS DE LA VIA BILIAR, RESOLUCION Y SEGUIMIENTO. 85 Congreso Argentino de Cirugía. Buenos Aires.

9. Juan Calderón Reza, L. V. (2016). IATROGENIA DE VÍAS BILIARES, RECONSTRUCCIÓN QUIRÚRGICA BILIODIGESTIVA, REPORTE DE UN CASO. Revista Científica y Tecnológica UPSE, III, 38-39.

10. Juan José Granados R, A. G. (2016). Current perspective in the treatment of bile duct injuries. (M. Academy, Ed.) International Journal of Research in Medical Sciences, 4, 677-684.

11. Klaske A.C. Booij, R. J. (2018). Seguimiento a largo plazo y factores de riesgo de estenosis después de la hepaticoyeyunostomía por lesión del conducto biliar: un análisis del tratamiento quirúrgico y percutáneo en un centro terciario. Surgery in Practice and Science, 7. doi: https://doi.org/10.1016/j.surg.2018.06.038

12. López, D. A. (2015). Derivación bilioentérica en un centro académico de referencia estatal en México. medigraphic.com/Cirujanogeneral, 9. Obtenido de https://www.medigraphic.com/pdfs/cirgen/cg-2015/cg153_4c.pdf 
ISSN: 2600-5859

13. Mariano Palermo, D. B. (2012). Derivaciones Biliodigestivas por vía Laparoscópica. En CLÍNICA QUIRÚRGICA (págs. 67-91). Buenos Aires.

14. Martin D1, U. E. (2016). Bile duct injuries after laparoscopic cholecystectomy: 11-year experience in a tertiary center. PubMed, 197-201.

15. Martín Martín Ernesto, G. P. (2015). Lesiones iatrogénicas de conductos biliares aberrantes tras Colecistectomía. Cirugía Española, 470.

16. Óscar Chapa-Azuela, 1. V.-H.-D.-M. (2013). Tratamiento quirúrgico de las lesiones yatrógenas de la vía biliar. Revista Médica del Hospital General de México., 76, 7-14.

17. Straka M, H. E. (2017). Iatrogenic biliary tract lesions requiring surgical reconstruction presentation and classification of the lesions, their reconstruction and evaluation of the results. PubMed, 9-17.

18. Townsend, C. -B.-E.-M. (2013). Sabiston. Tratado de Cirugía. (19 ed.). Madrid-España: Elsevier.

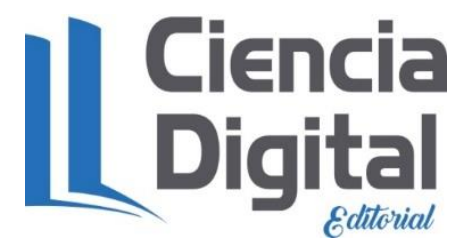




\section{PARA CITAR EL ARTÍCULO INDEXADO}

León Loaiza, F. K., \& Andrade Astudillo, D. M. (2020). Estenosis de Anastomosis Hepático-yeyunal TerminoLateral en $Y$ de Roux tratada mediante reconfección y modificación de la técnica de Anastomosis a Termino-Terminal. ConcienciaDigital, 3(1.1), 284-295.

https://doi.org/10.33262/concienciadigital.v3i1.1.1148

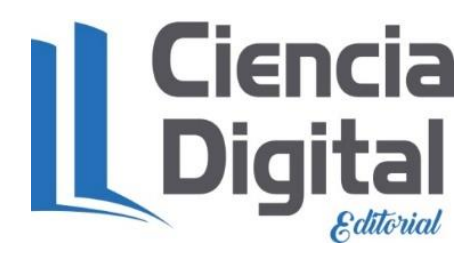

El artículo que se publica es de exclusiva responsabilidad de los autores y no necesariamente reflejan el pensamiento de la Revista Conciencia Digital.

El artículo queda en propiedad de la revista y, por tanto, su publicación parcial y/o total en otro medio tiene que ser autorizado por el director de la Revista Conciencia Digital.
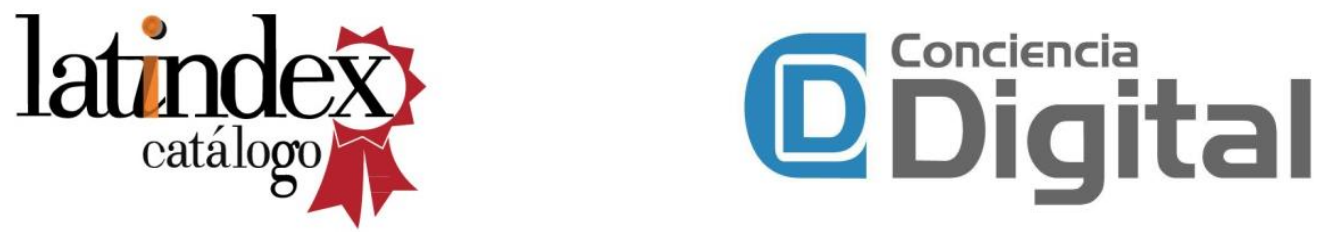